\title{
Holographic optical elements for the extreme-ultraviolet regime
}

\author{
Patrick P. Naulleau, Farhad Salmassi, Eric M. Gullikson, and Erik H. Anderson \\ Center for X-Ray Optics, Lawrence Berkeley National Laboratory, Berkeley, CA 94720
}

\begin{abstract}
As the development of extreme ultraviolet (EUV) lithography progresses, interest grows in the extension of traditional optical components to the EUV regime. The strong absorption of EUV by most materials and its extremely short wavelength, however, makes it very difficult to implement many components that are commonplace in the longer wavelength regimes. One such component is the diffractive optical element used, for example, in illumination systems to efficiently generate modified pupil fills. Here we demonstrate the fabrication and characterization of EUV binary phase-only computer-generated holograms allowing arbitrary far-field diffraction patterns to be generated.
\end{abstract}

Keywords: extreme ultraviolet, holography, diffraction, holographic-optical element

\section{INTRODUCTION}

Extreme ultraviolet (EUV) lithography [1] remains a top candidate for volume production at the 32-nm generation of nano-electronics. Development of this technology has sparked interest in the extension of a variety of optical elements to the EUV regime. Examples of such elements include gratings, diffusers, and diffractive or holographic optical elements. The fabrication of high efficiency EUV gratings [2] and diffusers [3] using reflective relief structures has already been demonstrated. Here we apply similar approaches to the fabrication of an EUV binary phase-only computer-generated hologram. Moreover, we consider several other fabrication approaches and demonstrate the fabrication of a null-hologram (grating) using an etched-multilayer technique.

Diffractive or holographic optical elements can play an important role in lithography systems by providing an efficient mechanism for generating modified illumination. As first proposed by Kamon et al., modified illumination schemes are cost-effective mechanisms for resolution and depth-of-focus enhancement [4]. Often such illumination systems are produced by placing a variable aperture in the pupil plane of the illuminator. This method, however, comes at the cost of optical throughput. Noting that source power is already an issue for EUV systems, incurring further optical losses through the use of apertures in the illuminator may not be a feasible solution. For example, in the commercial microfield EUV exposure tool installed at SEMATECH North, utilizing the aperture-based dipole illumination setting would come at the cost of a $97 \%$ loss in throughput [5]. The diffractive solution as presented by Himel et al. for visible and UV lithography systems, provides a mechanism for greatly enhanced throughput [6]. Here we present the extension of the diffractive approach to the EUV regime.

With the patterned relief approach we have demonstrated a generalized EUV holographic optical element (HOE) capable of an absolute efficiency into one diffracted order of $22 \%$. Using a binary carrier, the device produces diffracted orders that are nearly symmetric about the zero order meaning that in the case where axially symmetric diffraction patterns are desired, both positive and negative diffraction orders can be used, and the efficiency can be twice as high. So for dipole illumination, the device would have an efficiency of $44 \%, 15$ times higher than the $3 \%$ efficiency of the aperture-based method. For the etched-multilayer null hologram, we have demonstrated an absolute efficiency of $19 \%$ into the first diffracted order. The device we fabricated remains hampered by a residual absorbing layer in the phase shifted regions. Upon removal of the residual layer, the efficiency would be increased to $23 \%$. Again, the efficiency could be doubled for symmetric diffraction patterns. 


\section{EUV HOLOGRAM ARCHITECTURE}

A variety of architectures are possible for the fabrication of EUV holograms (Fig. 1). Efficiency being of primary concern in the EUV regime, we consider only phase devices. Phase holograms in the EUV can be made both as reflection and transmission devices. Transmission devices need to be fabricated onto thin membranes to support the phase-shifting material, which is ideally Molybdenum (Mo) [7]. An 86-nm thick layer of Mo provides a phase shift of $180^{\circ}$ and still has an intensity transmittance of $60 \%$. An ideal choice for the membrane is Silicon. Assuming a membrane thickness of $180 \mathrm{~nm}$, the transmission can be as high as $74 \%$. Ignoring the membrane, a Mo device can support an absolute efficiency of $32 \%$ into the first order. Further considering the membrane, the absolute efficiency becomes $24 \%$. As we will see below, this compares well with the achievable absolute efficiency from reflection devices.

Reflection HOEs can themselves take on several forms, including a patterned relief substrate overcoated with a multilayer reflector [8], an etched multilayer relying on the difference in index of refraction between vacuum and the multilayer [9], as well as a patterned Mo coating on top of the multilayer. In this case the Mo coating, the layer need only be $43-\mathrm{nm}$ thick owing to the round trip propagation through the material. Note that the latter two approaches are actually quite similar. As for the membrane case above, the reflector efficiency limits the absolute efficiency of the device. For EUV, an ideal multilayer would have a reflectivity as high as $73 \%$, however, in practice we could expect reflectivities only as high as approximately $67 \%$, rendering the multilayer case slightly worse than the Silicon membrane case considered above.

For the overcoated relief substrate case, the carrier can indeed be pure phase, yielding an ideal absolute efficiency of $27 \%$, assuming a presently achievable multilayer reflectivity of $67 \%$. This same efficiency can, in principle, be obtained using the etched multilayer configuration assuming the etched portion of the multilayer to still be comprised of at least 40 layer pairs. Finally, for the patterned Mo layer case, the ideal absolute efficiency becomes $22 \%$. This is slightly lower than the transmission case since the assumed multilayer reflectivity is lower than the membrane transmittance assumed above.

The calculation results show the various methods to have nearly the same ideal performance. In practice, however, one method might be preferable over another in terms of efficiency if the HOE could be integrated into an otherwise needed optical element. For example, if the optical system contained a membrane used as a vacuum window or spectral filter, the HOE could be added directly to that device incurring no additional membrane losses. This, of course, assumes the location of the existing membrane to

(a)

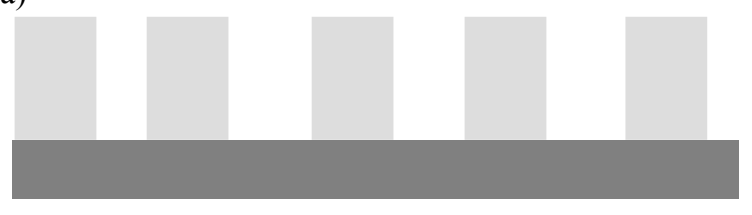

(b)

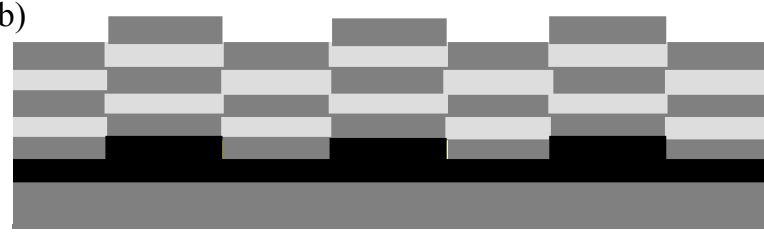

(c)

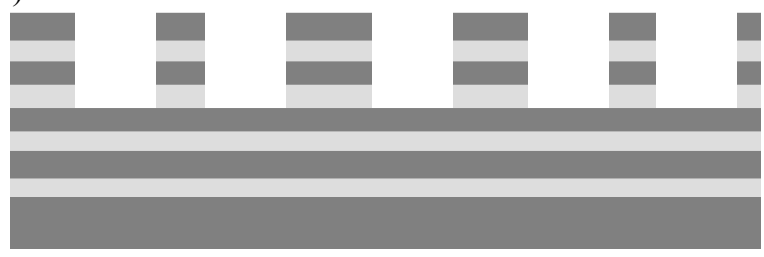

(d)

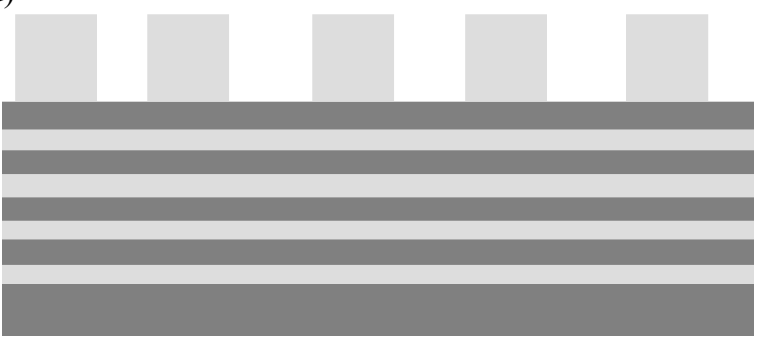

Fig. 1. Schematics of possible architectures for the realization of phase-carrier EUV holograms. (a) transmission device with 86-nm Mo phase-shifter on transparent membrane. (b) geometric phase shifter realized by overcoating relief structure with multilayer reflector. (c) Etched multilayer, the phase shifter is the protruding multilayer itself. (d) $43-\mathrm{nm}$ Mo phase-shifter on top of multilayer reflector. To aid in the visualization, only a few layer pairs are shown. In practice there would be approximately 40 layer pairs, with at lease 20 pairs remaining in the etched area for (c). 
be compatible with the requirements of the HOE to be added to the system. A similar argument can be made for the reflection $\mathrm{HOE}$ case by adding the HOE to an existing multilayer mirror.

\section{HOLOGRAM SYNTHESIS}

As described above, efficiency is of primary concern for EUV holograms, especially if one intends to use the device for lithography or microscopy. Restricting ourselves to a binary carrier, the optimal diffraction efficiency is obtained using a $50 \%$ duty-cycle carrier. Forcing the binary carrier to maintain a duty cycle of $50 \%$ implies that amplitude modulation of the wavefront is not possible, we can thus only use phase modulation. Note that in general the phase of the generated wavefront will be controlled by the placement of the lines on the hologram (phase of the carrier) and the amplitude would be controlled by duty cycle, locally deviating from $50 \%$ will serve to locally attenuate the diffracted beam.

The holograms we consider here are of the Fourier Transform type [10]. Being restricted to pure phase modulation of the wavefront, we cannot simply Fourier transform the desired far field pattern to determine the modulating function since the Fourier transform will, in general, yield a complex-valued function. To get around this problem an iterative approach can be used to calculate the hologram modulating function. When calculating the modulating function for a phase-only HOE intended to produce a specific diffraction pattern, two parameters are known: 1) the magnitude of the Fourier transform of the modulating function (the desired diffraction pattern) and 2) the amplitude of the modulating function (unity because it is a phase-only device). The problem is, thus, to determine the phase of the HOE modulating function given the amplitude of its Fourier transform.

The phase-only modulation function problem is similar to the astronomical problem of reconstructing an object given the magnitude of the Fourier transform of the object. To address this problem, several so-called phase retrieval algorithms have been developed based on iterative techniques $[11,12]$. The implementation of the iterative technique we use here is the error-reduction method. The result of this iterative process is to generate a two-dimensional quasicontinuous pure phase function $[\Phi(x, y)]$ whose diffraction pattern closely matches the desired diffraction pattern. To achieve adequately high accuracy encoding of this pure phase function, spatial carrier encoding is required, hence the need for an HOE. As our spatial carrier we choose a square-wave phase grating. Using a simpler amplitude carrier would also be possible; however, this would come at the cost of much reduced efficiency.

For the example presented here, we choose a carrier period of $392 \mathrm{~nm}$, which at a wavelength of $13.5-\mathrm{nm}$ yields a carrier propagation angle of $2^{\circ}$. The numerical aperture (half angle) of the first order diffraction pattern (the LBNL logo) is set to approximately $0.042 \times 0.026$, the narrow width being in the carrier direction. For computational efficiency, we generate the HOE over a $200 \times 200 \mu \mathrm{m}$ area comprised of $2048 \times 2048$ pixels. Each computed pixel corresponds to a $98 \times 98 \mathrm{~nm}$ square area in the final device. The full patterned hologram is then comprised of a series of these areas stitched together to cover a $1 \mathrm{~mm}^{2}$ area (Fig. 2). Figure 2 also shows one of these $200 \times 200 \mu \mathrm{m}$ areas along with an expanded view of a $20 \times 20 \mu \mathrm{m}$ area. White regions represent areas of $\pi$ phase shift and black regions zero phase shift. The fidelity of the computed phaseonly HOE can be evaluated by simply Fourier

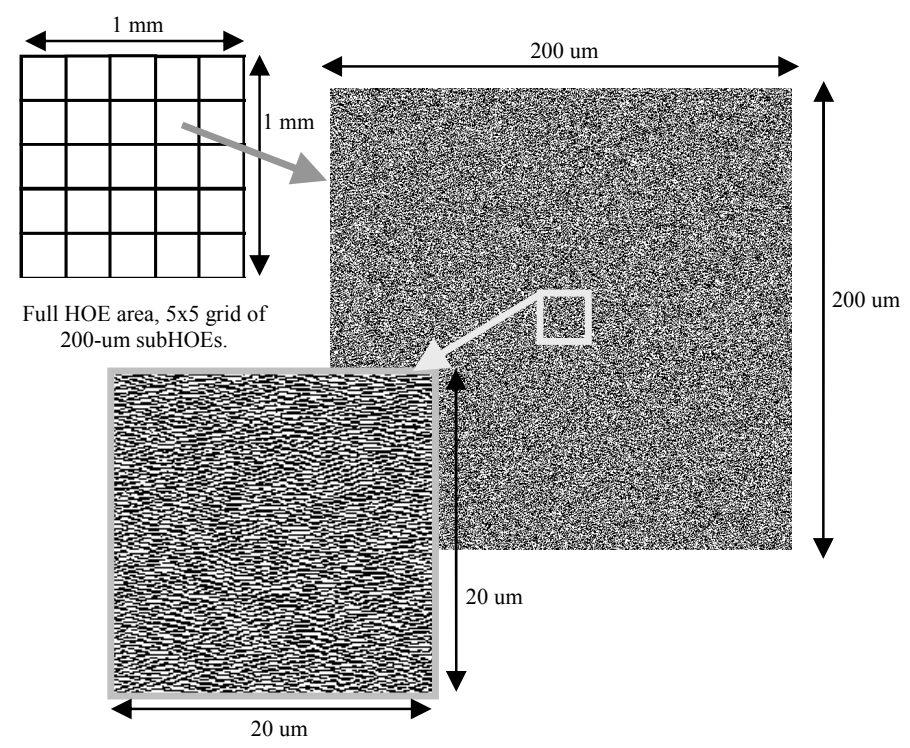

Fig. 2. Schematic of $\mathrm{HOE}$ area comprised of a $5 \times 5$ grid of subfields. HOE is computed over a $200 \times 200 \mu \mathrm{m}$ area comprised of $2048 \times 2048$ pixels forming a subfield replicated across the desired HOE area. Each computed pixel corresponds to a $98 \times 98 \mathrm{~nm}$ square area in the final device. Black and white regions in the depictions of the computed HOE represent areas of zero and $\pi$ phase shift respectively. 
transforming the computed HOE and extracting the first diffracted order. Doing so yields the computed far-field intensity pattern shown in Fig. 3. As expected, the diffraction pattern closely resembles the target LBNL logo despite the HOE being a pure phase device with pure phase modulation of the diffracted wavefront.

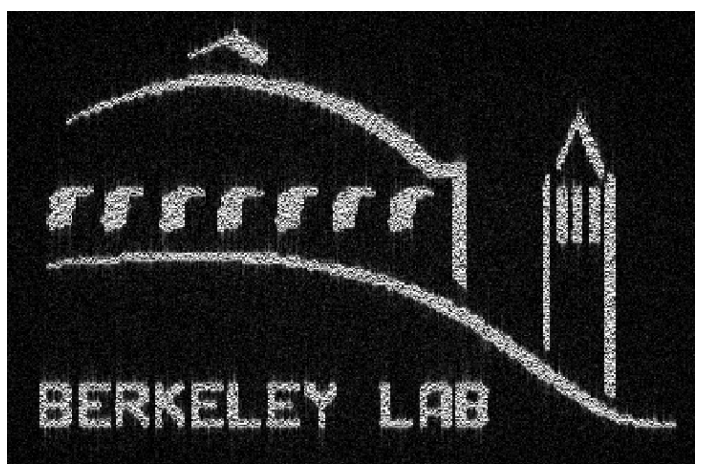

Fig. 3. Fourier transform of the computed HOE subfield from Fig. 2. The area corresponding to the first diffracted order is shown, demonstrating the fidelity of the computed binary phase-only hologram. The computed diffraction pattern differs from the target only by virtue of the fine speckle pattern.

\section{RELIEF HOLOGRAM FABRICATION AND TESTING}

The first EUV hologram we fabricated used the relief substrate architecture and was fabricated using a hardmask method previously described [13]. The HOE was characterized at the calibration and standards bend-magnet beamline 6.3.2 at the Advanced Light Source located at Lawrence Berkeley National Laboratory [14]. The measurements were performed with a spectral resolution, $\lambda / \Delta \lambda$, of approximately 1400 . The HOE was characterized by illuminating it with a low-divergence beam and placing an imaging detector in the far field to record the diffraction pattern (Fig. 4). Owing to the geometry of the measurement, it was not possible to record the full extent of the diffraction pattern in a single exposure. The image in Fig. 4 is actually a composite of several sub-images stitched together.

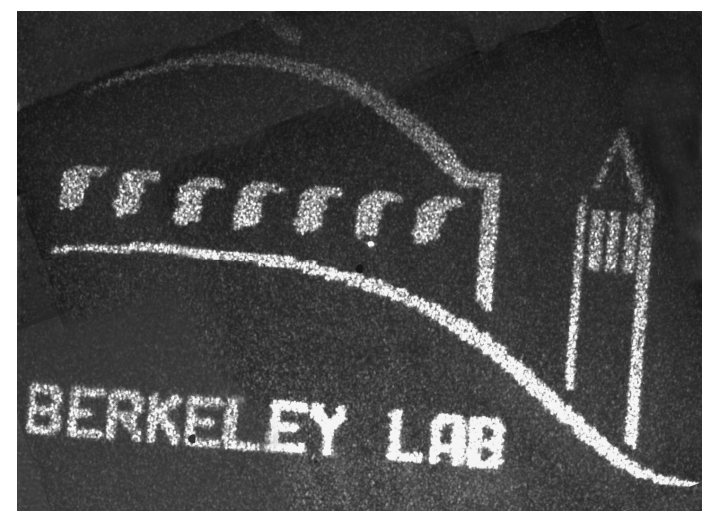

Fig. 4. At-wavelength $(13.5 \mathrm{~nm})$ characterization of the fabricated HOE. An imaging detector is used to record the far-field diffraction pattern under low-divergence illumination. Owing to the geometry of the system, it was not possible to record the full extent of the first-order diffraction pattern in a single exposure. The displayed image is a results of stitching several recorded images.

Geometric limitations of the reflectometer prevent us from measuring the absolute efficiency of the actual hologram. This is due to the large NA of the holographic image relative to the collection NA of the detectors. In lieu of characterizing the absolute efficiency hologram presented above, we used the reflectometer to characterize a null hologram (carrier only). Detailed description of this measurement can be found in Ref. [13]. We choose the carrier period of the null hologram to be 200-nm, nearly half the size of the carrier used for the HOE presented here. Choosing a smaller period corresponds to a more stringent condition due to the multilayer smoothing. Characterizing the efficiency of the comparable grating yields an absolute first-order efficiency of $22 \%$. Normalizing this result to the multilayer reflectivity of $65 \%$ yields an effective diffraction efficiency of $35 \%$. These results compare well to the theoretical limits of $27 \%$ (assuming $67 \%$ multilayer reflectivity) for the absolute efficiency and $40 \%$ for the normalized efficiency. 


\section{ETCHED MULTILAYER NULL HOLOGRAM}

Although extremely efficient, a significant drawback of the overcoated relief pattern architecture is limited pattern resolution (diffraction NA) due to the multilayer smoothing effect [15]. In practice, this effect will limit the relief pattern to feature sizes of $100-\mathrm{nm}$ and larger. This problem can be avoided, however, if we choose one of the patterned phase-shifter approaches. As described above, the approaches includes patterned Mo on a membrane or reflector as well as etched multilayer. The patterned Mo approach has been demonstrated in the past [7] at least for the null-hologram case of a simple grating, however, etch resolution improvements are still required in order to achieve adequate resolution and efficiency. We continue to pursue improvements in this area.

The multilayer etch method also shows considerable promise. This method has been used in the past to fabricate phase-shift masks for EUV [9], however, the performance had been limited by sidewall slope issues. A new etch process has been developed at LBNL largely alleviating this problem. Figure 5 shows a scanning electron micrograph of a recently fabricated etched multilayer grating with $500-\mathrm{nm}$ period. An embedded chrome etch-stop layer is used to control the etch depth. The high resolution of the process is evident. For this initial demonstration, the etch depth target was $115 \mathrm{~nm}$ in order to achieve a phase shift of approximately $180^{\circ}$.

Figure 6 shows the characterization results for the null hologram. A diffraction efficiency of $19 \%$ into the first diffracted orders is measured. We also observe strong suppression of the zero-order term. We note that the reflectivity clear multilayer was measured to be only $55 \%$, considerably lower than the target value of $67 \%$ or higher. The lower reflectivity is due to a residual hardmask layer on top of the multilayer used in the patterning process. Improving the hardmask strip could expect the efficiency to improve to $23 \%$. Further removing the residual absorbing chrome layer in the etched regions, the efficiency would be expected to reach the theoretical limit of $27 \%$ assuming a multilayer reflectivity of $67 \%$.

\section{SUMMARY}

Various potential architectures, including both transmission and reflection, for the realization of EUV HOE's have been presented. With efficiency being of utmost concern, we have concentrated on pure phase devices both from the perspective of the carrier as well as the modulating signal. The various methods support absolute efficiencies exceeding $20 \%$ into the first order. The highest theoretical efficiency method is the patterned relief substrate overcoated with multilayer. Using this method, an HOE with an absolute efficiency of $22 \%$ at a carrier period of $200 \mathrm{~nm}$ has been

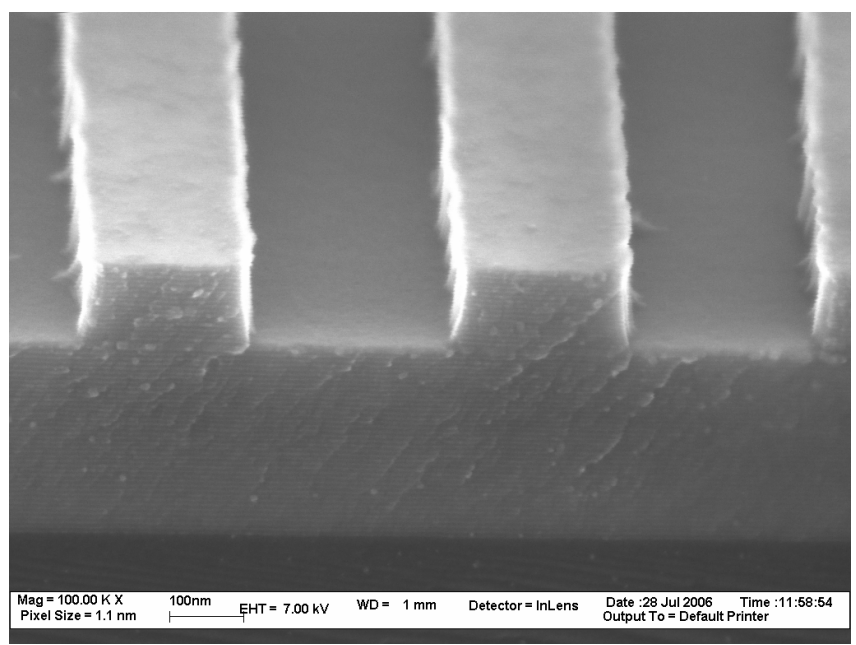

Fig. 5. Scanning electron micrograph of an etched multilayer grating with 500-nm period. An embedded chrome etchstop layer is used to control the etch depth. For this demonstration, the etch depth is set to approximately 115 $\mathrm{nm}$, yielding a phase shift of $180^{\circ}$.

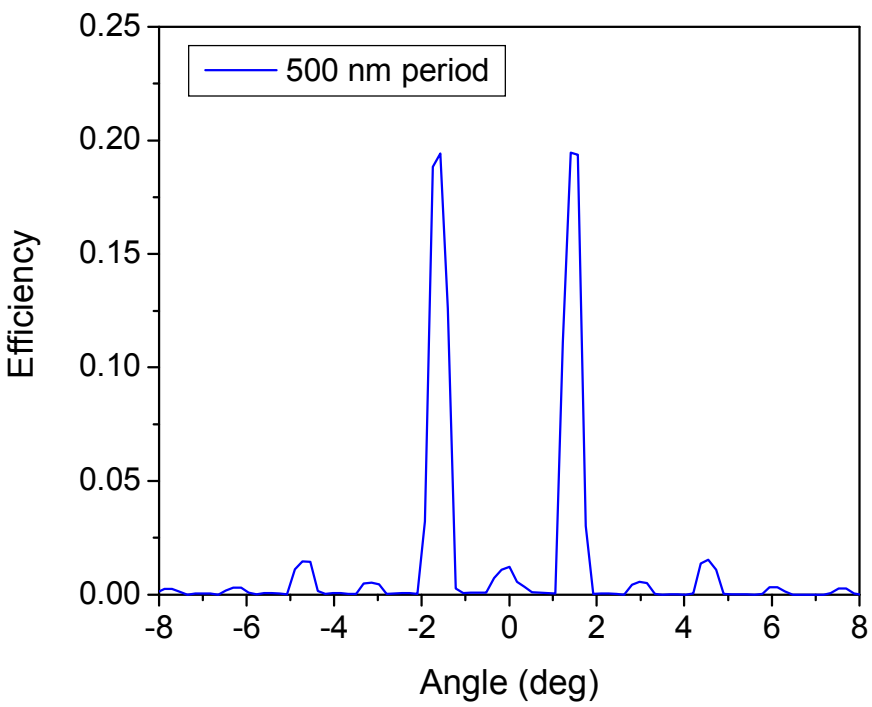

Fig. 6. Characterization results for the etched-multilayer null hologram in Fig. 5. First order absolute diffraction efficiency is $19 \%$. The as-deposited multilayer was measured to have a reflectivity of $55 \%$. 
demonstrated. A drawback of this method, however, is the limited resolution due to the multilayer smoothing effect. To address this concern we also pursued the etched multilayer approach. Using this method the feasibility of a highresolution $\mathrm{HOE}$ has been demonstrated through the fabrication of a $500-\mathrm{nm}$ period grating. Near theoretical performance was achieved with this device as well, however, further improvements will require the optimization of the strip of the hardmask and chrome etch-stop.

The authors are greatly indebted to Bruce Harteneck and Eugene Veklerov for expert programming and fabrication support, to Andrew Aquila for EUV metrology support, and to the entire CXRO staff for enabling this research. This research was performed at Lawrence Berkeley National Laboratory, which is operated under the auspices of the Director, Office of Science, Office of Basic Energy Science, of the US Department of Energy.

\section{REFERENCES}

1. R. Stulen and D. Sweeney, "Extreme ultraviolet lithography," IEEE J. Quantum Electron. 35, 694-699 (1999).

2. P. Naulleau, J. Liddle, E. Anderson, E. Gullikson, P. Mirkarimi, F. Salmassi, and E. Spiller, "Fabrication of highefficiency multilayer-coated gratings for the EUV regime using e-beam patterned substrates," Opt. Comm. 229, 109-116 (2003).

3. P. Naulleau, J. Liddle, F. Salmassi, E. Anderson, E. Gullikson, "Design, fabrication, and characterization of highefficiency extreme ultraviolet diffusers," Appl. Opt. 43, 5323-5329 (2004).

4. K. Kamon, T. Miyamoto, Y. Myoi, H. Nagata, M. Tanaka, K. Horie, "Photolithography system using annular illumination," Japanese Journal of Applied Physics 30, 3021-3029 (1991).

5. P. Naulleau, K. Dean, and K. Lowack, "Aerial-image modeling for the extreme ultraviolet microfield exposure tool at SEMATECH North,” Proc. SPIE 6151, 6151098 (2006).

6. M. Himel, R. Hutchins, J. Colvin, M. Poutous, A. Kathman, A. Fedor, "Design and fabrication of customized illumination patterns for low k1 lithography: a diffractive approach," Proc. SPIE 4346, 1436-1442 (2001).

7. P. Naulleau, C. Cho, E. Gullikson, and J. Bokor, "Transmission phase gratings for EUV interferometry," J. Synch. Rad. 7, 405-410 (2000).

8. F. Salmassi, P. P. Naulleau, E. M. Gullikson, D. L. Olynick, and J. A. Liddle, "Extreme ultraviolet binary phase gratings: Fabrication and application to diffractive optics," J. Vac. Sci. \& Technol. A 24, 1136-1140 (2006).

9. B. La Fontaine, A. Pawloski, O. Wood, Y. Deng, H. Levinson, P. Denham, E. Gullikson, B. Hoef, P. Naulleau, C. Holfeld, C. Chovino, F. Letzkus, "Demonstration of phase-shift masks for extreme-ultraviolet lithography," Proc. SPIE 6151, 6151010 (2006).

10. Goodman Introduction to Fourier Optics $2^{\text {nd }}$ Ed., Chapt.

11. R. Gerchberg and W. Saxon, "A practical algorithm for the determination of phase from image and diffraction plane pictures," Optik 35, 237-246 (1972).

12. J. Fienup, "Reconstruction of an object from the modulus of its Fourier transform," Opt. Lett. 3, 27-29 (1978).

13. F. Salmassi, P. P. Naulleau, E. M. Gullikson, D. L. Olynick, and J. A. Liddle, "Extreme ultraviolet binary phase gratings: Fabrication and application to diffractive optics," J. Vac. Sci. \& Technol. A 24, 1136-1140 (2006).

14. J. H. Underwood, E. M. Gullikson, M. Koike, P. J. Batson, P. E. Denham, K. D. Franck, R. E. Tackaberry, and W. F. Steele, Rev. Sci. Instrum. 9, 1-5 (1996).

15. E. Spiller, S. L. Baker, P. B. Mirkarimi, V. Sperry, E. M. Gullikson, and D. G. Stearns, "High-Performance Mo-Si Multilayer Coatings for Extreme-Ultraviolet Lithography by Ion-Beam Deposition ," Appl. Opt. 42, 4049-4058 (2003). 